\title{
Characterization of Electron Mediator Preference of Aerococcus viridans-Derived Lactate Oxidase for Use in Disposable Enzyme Sensor Strips
}

\author{
Noya Loew, ${ }^{\dagger}$ Maya Fitriana, ${ }^{\dagger}$ Kentaro Hiraka, Koji Sode, ${ }^{1}$ and Wakako Tsugawa ${ }^{*}$ \\ Department of Biotechnology and Life Science, Graduate School of Engineering, \\ Tokyo University of Agriculture and Technology, \\ 2-24-16 Nakacho, Koganei, Tokyo 184-8588, Japan \\ ${ }^{1}$ Institute of Global Innovation Research, Tokyo University of Agriculture and Technology, \\ 3-8-1 Harumi-cho, Fuchu, Tokyo 184-8538, Japan
}

(Received August 30, 2017; accepted December 7, 2017)

Keywords: lactate oxidase, screen-printed carbon electrode, electron mediator, 1-methoxy-5-methyl phenazinium methyl sulfate, potassium ferricyanide, hexaammineruthenium(III) chloride, biomedical engineering

Monitoring blood lactate concentration is important in clinical diagnosis and evaluation of fitness care. Electrochemical enzyme sensors are commonly used for the measurement of lactate owing to their simplicity and sensitivity. Lactate oxidases (LOxs) are widely used for the construction of lactate enzyme sensors, especially the LOx derived from Aerococcus viridans $(A v \mathrm{LOx})$. To minimize interference from redox substances, most lactate sensors utilize artificial mediators. However, reports on the comparison of different mediators in lactate measurements are limited. In this study, the preferences of $A v \mathrm{LOx}$ for electron mediators were investigated electrochemically. We concluded that the charge of the mediator plays a significant role in determining its suitability as a mediator for $A v$ LOx. The relatively uncharged 1-methoxy-5-methyl phenazinium methylsulfate (mPMS) was the preferred mediator for $A v \mathrm{LOx}$, the strongly negative ferricyanide was a less preferential mediator, and the strongly positive hexaammineruthenium(III) was not utilized as a mediator by $A v \mathrm{LO}$. This suggests that the protein scaffold of $A v \mathrm{LOx}$ can almost completely block the access to the co-factor for very small electron acceptors, depending on their charge.

\section{Introduction}

Determination of the blood lactate concentration level is widely used in clinical diagnosis and evaluation of exercise in sports medicine. ${ }^{(1,2)}$ The normal blood lactate level in people at rest is $0.5-1.8 \mathrm{mM}^{(1)}$ An increased lactate level is indicative of hypoxia due to medical problems or exercise. $^{(1,2)}$ With extreme exercise, the lactate level can increase to up to $25 \mathrm{mM}^{(2)}$ Commonly, handheld blood lactate analyzers are used for the measurement of capillary blood lactate. ${ }^{(1)}$ These analyzers play a major role in lactate monitoring in the biomedical engineering field. The analyzers are based on amperometry and measure electric current generated by lactate oxidation using the enzyme lactate oxidase (LOx). LOx is a flavoenzyme that harbors flavin mononucleotide

\footnotetext{
*Corresponding author: e-mail: tsugawa@cc.tuat.ac.jp

$\dagger$ These authors contributed equally to this work.

http://dx.doi.org/10.18494/SAM.2017.1731
} 
(FMN) as the cofactor, and catalyzes the oxidation of lactate to pyruvate while flavin is reduced (in the reductive half-reaction). ${ }^{(3,4)}$ In the subsequent oxidative half-reaction, the reduced flavin is re-oxidized by oxygen, resulting in oxidized cofactor and hydrogen peroxide. ${ }^{(3,4)}$ Alternatively, electrons can be transferred from the reduced flavin in LOx to artificial electron mediators.

Therefore, second-generation lactate sensors utilizing LOx can be developed, which employ artificial electron mediators to avoid the interference of redox substances in the blood by allowing the application of a lower potential than that necessary to oxidize hydrogen peroxide.

To date, many artificial mediators for LOx sensors have been reported, such as ferrocene derivatives, ${ }^{(5-11)}$ indophenol derivatives, ${ }^{(5,7)}$ indoaniline derivatives, ${ }^{(10,11)}$ phenoxazine derivatives (i.e., Meldola blue) ${ }^{(6)}$ tetrathiafulvalene, ${ }^{(12)}$ hydroquinone, ${ }^{(5)}$ and metal complexes including ferricyanide, ${ }^{(5)} \mathrm{Ni}(\mathrm{II})$-cyclam, ${ }^{(5)}$ and Os complexes. ${ }^{(7,11,13)}$ Some reports compared different mediators regarding their suitability for LOx-based lactate sensors. ${ }^{(5,6,8,11,13)}$ However, only few papers discussed influential factors other than the local mediator concentration at the electrode/ solution interface. ${ }^{(5,11,13)}$

One of the most common artificial electron mediators used in commercially available enzyme sensors is potassium ferricyanide. Ferricyanide is a small, water-soluble, inorganic metal complex with reversible electron transfer kinetics. However, ferricyanide is easily reduced by light irradiation, which leads to measurements that are too high. Recently, hexaammineruthenium(III) chloride has been attracting attention as a mediator because it allows for a lower application potential and because it is more stable under light irradiation than ferricyanide. Hexaammineruthenium(III) is also a small, water-soluble, inorganic metal complex with reversible electron transfer kinetics. Although these mediators are commonly used, there is no report that compares these mediators scientifically.

In this paper, we compare the commonly used electron mediators ferricyanide, hexaammineruthenium(III), and 1-methoxy-5-methyl phenazinium methylsulfate (mPMS; the electroactive form of phenazinium methylsulfate, an electron acceptor often used in spectrophotometric assays) in combination with Aerococcus viridans-derived LOx (AvLOx). We use end-point assays with disposable film electrodes mimicking enzyme sensor strips and discuss the preference of $A v \mathrm{LOx}$ for these mediators.

\section{Materials and Methods}

\subsection{Materials and apparatus}

2,6-dichlorophenolindophenol (DCIP) was purchased from Merck (Darmstadt, Germany). 4-Aminoantipyrine (4AA), 5-methyl-phenazinium methylsulfate (PMS), and potassium ferricyanide $\left\{\mathrm{K}_{3}\left[\mathrm{Fe}(\mathrm{CN})_{6}\right]\right\}$ were purchased from Kanto Chemical (Tokyo, Japan). 3-(N-ethyl3-methylanilino)-2-hydroxy-propanesulfonate (TOOS) and mPMS were purchased from Dojindo Laboratories (Kumamoto, Japan). Peroxidase (POD) was obtained from Amano Enzyme (Gifu, Japan). Sodium L-lactate and hexaammineruthenium(III) chloride $\left\{\left[\mathrm{Ru}\left(\mathrm{NH}_{3}\right)_{6}\right] \mathrm{Cl}_{3}\right\}$ were purchased from Sigma-Aldrich (St. Louis, MO, USA).

Screen-printed carbon electrodes (SPCEs; working electrode: carbon $2.4 \mathrm{~mm}^{2}$; reference electrode: $\mathrm{Ag} / \mathrm{AgCl}$; counter electrode: carbon) were kindly supplied by i-SENS (Seoul, Republic of Korea). All electrochemical measurements were carried out with a VersaSTAT4 potentiostat from Princeton Applied Research (AMETEC Inc., Berwyn, PA, USA). 


\subsection{Enzyme preparation}

$A v \mathrm{LOx}$ was prepared by recombinant expression in Escherichia coli. ${ }^{(3)}$ The $K_{m}$ of $A v \mathrm{LOx}$ was $0.61 \mathrm{mM}$ and the $V_{\max }$ was $101 \mathrm{U} / \mathrm{mg}$ based on the oxidase activity (Table 1), which are comparable to those in the literature. ${ }^{(3,4)}$ The dehydrogenase activity was $135 \mathrm{U} / \mathrm{mg}$ (Table 1 ).

\subsection{Enzyme activity}

The oxidase activity was measured using $1.5 \mathrm{mM}$ 4AA, $1.5 \mathrm{mM}$ TOOS, $2 \mathrm{U} / \mathrm{ml} \mathrm{POD}$, and 0-25 mM lactate in $10 \mathrm{mM}$ potassium phosphate buffer (PPB; $\mathrm{pH}$ 7.0). The enzymatic reaction was initiated by the addition of lactate. One unit (U) of enzyme activity was defined as the amount of enzyme that catalyzes the production of $1 \mu \mathrm{mol}$ of $\mathrm{H}_{2} \mathrm{O}_{2}$ per minute using $20 \mathrm{mM}$ lactate at $25^{\circ} \mathrm{C}$ (the absorbance change at $555 \mathrm{~nm}$ due to the formation of quinone imine dye was monitored). Dehydrogenase activity was measured using $4 \mathrm{mM}$ PMS, $0.06 \mathrm{mM}$ DCIP, and 0-25 $\mathrm{mM}$ lactate in $10 \mathrm{mM}$ PPB (pH 7.0). One unit (U) of enzyme activity was defined as the amount of enzyme that catalyzes the reduction of $1 \mu \mathrm{mol}$ DCIP per minute using $20 \mathrm{mM}$ lactate at $25^{\circ} \mathrm{C}$ (the absorbance change at $600 \mathrm{~nm}$ was monitored).

\subsection{Electrochemical measurements}

Chronoamperometry measurements were carried out. SPCEs were prepared by attaching a spacer and cover to form a $\mu \mathrm{L}$-volume capillary space above the electrodes. A premix $(4 \mu \mathrm{L})$ was prepared containing a mediator [ferricyanide, mPMS, or hexaammineruthenium(III)] and $A v \mathrm{LOx}$ in $100 \mathrm{mM}$ PPB (pH 7.0). The enzyme reaction was started by adding lactate $(1 \mu \mathrm{L})$ to the premix and $1.8 \mu \mathrm{L}$ of the mixture was loaded onto an SPCE. The final concentration of lactate was $0,0.5,1,2,5,10,20,30$, or $50 \mathrm{mM}$. The amount of enzyme was adjusted so that $0.1 \mathrm{U}$ was loaded onto the SPCE. A potential $[+400 \mathrm{mV}$ for ferricyanide, $+200 \mathrm{mV}$ for mPMS, $0 \mathrm{mV}$ for hexaammineruthenium(III)] was applied $60 \mathrm{~s}$ after the addition of lactate (the wait time) and the current was monitored for another $60 \mathrm{~s}$. For the standard curves, the current at $10 \mathrm{~s}$ (sampling time) after application of the potential was plotted against the lactate concentration.

\section{Results and Discussion}

\subsection{Electrochemical measurements with ferricyanide as mediator}

Electrochemical measurements were carried out, which mimicked blood lactate sensors. For these end-point assays, the enzyme, mediator and lactate were mixed before loading onto the

Table 1

Properties of purified $A v \mathrm{LO}$. Parameters determined from Hanes-Woolfs plots.

\begin{tabular}{lcc}
\hline & Oxidase activity & Dehydrogenase activity \\
\hline$K_{m}(\mathrm{mM})$ & 0.61 & 0.58 \\
$V_{\max }(\mathrm{U} / \mathrm{mg})$ & 101 & 135 \\
$V_{\max } / K_{m}(\mathrm{U} / \mathrm{mg} \cdot \mathrm{mM})$ & 167 & 232 \\
\hline
\end{tabular}


electrode strip. A potential was applied after a sufficient time to allow the complete conversion of the substrate (the wait time). Thus, the electrochemical measurement is that of a specific amount of reduced mediator, which reflects the initial amount of substrate. In these types of measurements, the current is described by the Cottrell equation, that is, the current is initially high but decreases with time.

Figure 1 shows the time courses of measurements with $100 \mathrm{mM}$ ferricyanide as a mediator and various concentrations of lactate. The amount of enzyme was chosen so that the complete conversion of the substrate was achieved within $60 \mathrm{~s}$ for all lactate concentrations. With increasing lactate concentration, the response current increased, indicating that a lactate sensor could be constructed with ferricyanide as the mediator. This was expected, as ferricyanide has been shown to be an electron acceptor for LOx. ${ }^{(5)}$

Corresponding measurements were carried out with other concentrations of ferricyanide, and the current at $10 \mathrm{~s}$ was plotted against the lactate concentration (Fig. 2). Without ferricyanide $(0 \mathrm{mM})$, no response current was observed, confirming that ferricyanide transfers electrons from the enzyme to the electrode. Similar sensitivities (slope of line; $0.64 \mu \mathrm{A} / \mathrm{mM}$ for $100 \mathrm{mM}$ ferricyanide) within the linear range were achieved with the different concentrations of ferricyanide, and the linear range extended to higher lactate concentrations for higher ferricyanide concentrations (Fig. 2). For each oxidation of one molecule of lactate, the LOx reduces two molecules of ferricyanide to two molecules of ferrocyanide. In this end-point assay, the enzyme reaction is finished before the potential is applied, which means that there are either no more lactate molecules or no more ferricyanide molecules. In the case of low initial lactate concentrations $(<20 \mathrm{mM})$, all lactate molecules are oxidized. The resulting amount of ferrocyanide molecules only depends on the initial lactate concentration, which is evidenced by the fact that the response currents do not depend on the ferricyanide concentration. When the initial lactate concentration exceeds half of the initial ferricyanide concentration, the amount of ferricyanide molecules becomes the limiting factor. This results in response currents depending on the ferricyanide concentration and not on the lactate concentration, and thus a loss of linearity. For high lactate

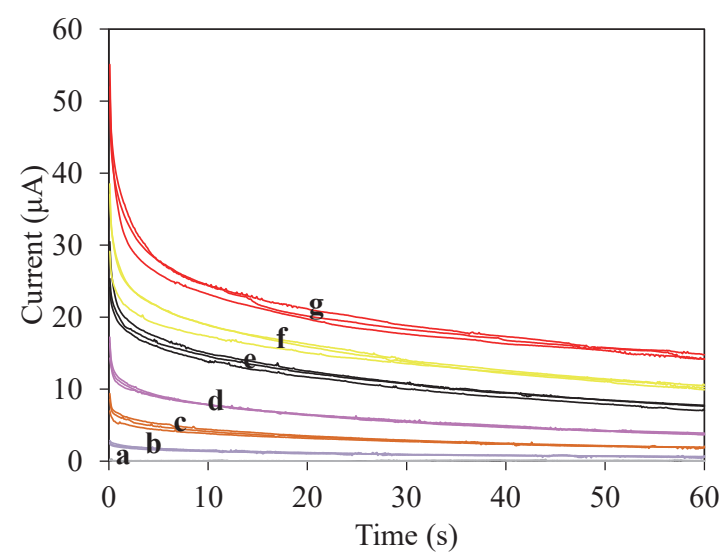

Fig. 1. (Color online) Time courses of response currents for various lactate concentrations. Mediator: $100 \mathrm{mM}$ ferricyanide; applied potential: $+400 \mathrm{mV}$; enzyme: $0.1 \mathrm{U}$ per sensor strip; wait time: $60 \mathrm{~s} ; n=3$. Lactate concentration: (a) 0, (b) 2, (c) 5, (d) 10, (e) 20, (f) 30, and (g) $50 \mathrm{mM}$. 


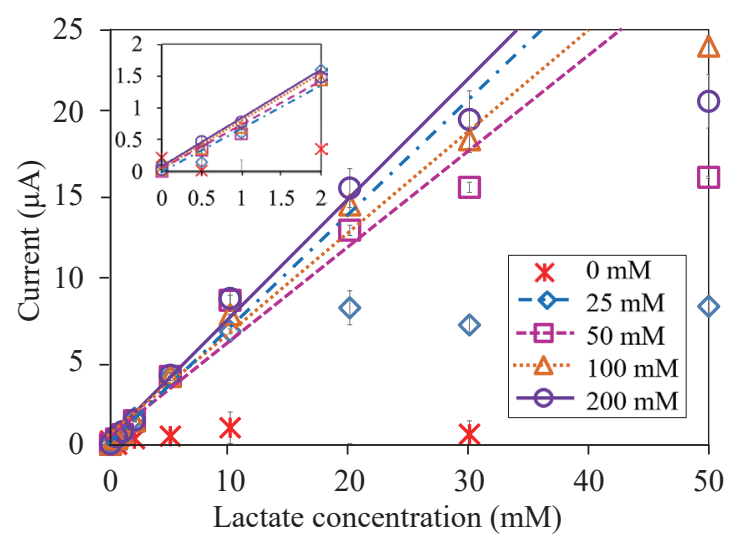

Fig. 2. (Color online) Standard curves of response currents vs lactate concentration for various ferricyanide concentrations. Wait time: $60 \mathrm{~s}$; enzyme: $0.1 \mathrm{U}$ per sensor strip; applied potential: $+400 \mathrm{mV}$; sampling time: $60 \mathrm{~s} ; n=3$. Inset: blow-up of low lactate concentrations.

concentrations $(50 \mathrm{mM})$, the obtained response currents were somewhat lower than expected for 100 and $200 \mathrm{mM}$ ferricyanide, indicating an incomplete turnover of lactate. A possible reason for this incomplete turnover is an insufficient amount of time. The time needed for the complete turnover of a given lactate amount (concentration) depends on the amount of enzyme. Therefore, further optimization (increasing) of the wait time or of the amount of $A v \mathrm{LOx}$ might lead to a wider linear range.

\subsection{Electrochemical measurements with different mediators}

Corresponding measurements to those described above for ferricyanide were carried out with mPMS and hexaammineruthenium(III) as mediators, and the currents at $10 \mathrm{~s}$ were plotted against the lactate concentration (Figs. 3 and 4). The properties of the mediators used in this study are summarized in Table 2.

Ferricyanide is a small inorganic compound and has a strong negative charge and a redox potential of $+0.23 \mathrm{~V}$ vs $\mathrm{Ag} / \mathrm{AgCl}{ }^{(14)}$ Hexaammineruthenium(III) is also a small inorganic compound, but with a strong positive charge and a redox potential of $-0.11 \mathrm{~V}$ vs $\mathrm{Ag} / \mathrm{AgCl}{ }^{(15)}$ The third mediator used in this study, mPMS, is an organic compound with either a weak positive charge (oxidized form) or no charge (reduced form), and a similar redox potential to hexaammineruthenium(III) $(-0.14 \mathrm{~V}$ vs $\mathrm{Ag} / \mathrm{AgCl}) .{ }^{(16)}$

With mPMS as a mediator, which is used as one mediator in a commercial sensor based on LOx, ${ }^{(17)}$ higher currents were achieved than with ferricyanide (Fig. 3). In contrast to ferricyanide, the LOx reduces only one molecule of mPMS for each oxidation of one molecule of lactate. Therefore, less mPMS is needed and a wide linear range can be achieved with lower mPMS concentrations. Furthermore, the wait time was long enough for the complete turnover of up to $50 \mathrm{mM}$ lactate, indicating that the electron transfer from the FMN of $A v \mathrm{LOx}$ to mPMS was faster than that to ferricyanide. However, the background current was high and increased with increasing mPMS concentration. This indicates that the mPMS used contained some interfering substance 


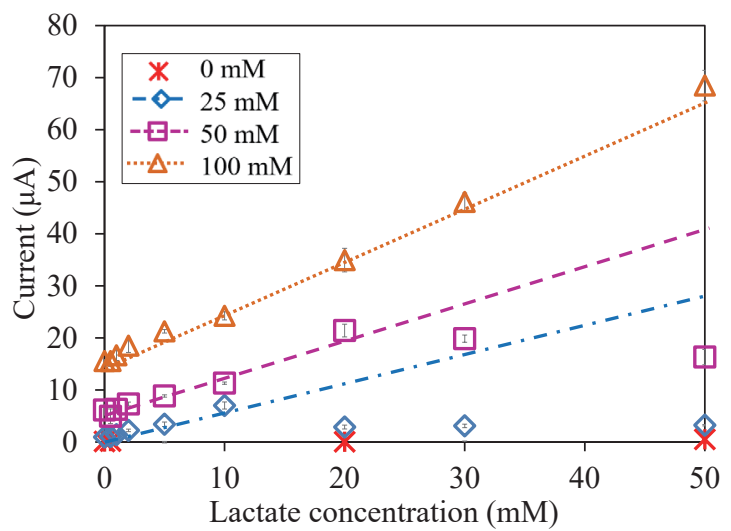

Fig. 3. (Color online) Standard curves of response currents vs lactate concentration for various mPMS concentrations. Wait time: $60 \mathrm{~s}$; enzyme: $0.1 \mathrm{U}$ per sensor strip; applied potential: $+200 \mathrm{mV}$; sampling time: $10 \mathrm{~s} ; n=3$.

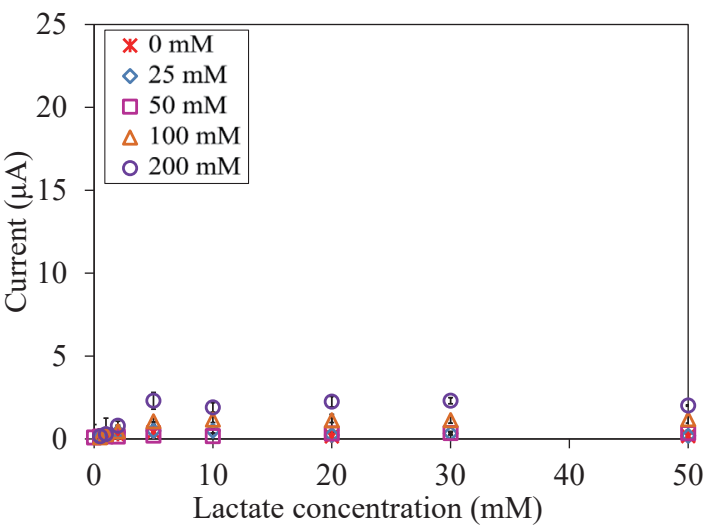

Fig. 4. (Color online) Standard curves of response currents vs lactate concentration for various hexaammineruthenium(III) concentrations. Wait time: $60 \mathrm{~s}$; enzyme: $0.1 \mathrm{U}$ per sensor strip; applied potential: $0 \mathrm{mV}$; sampling time: $10 \mathrm{~s} ; n=3$.

Table 2

Properties of mediators used in this study. All mediators are water soluble.

\begin{tabular}{|c|c|c|c|c|}
\hline Electron mediator & Type & $\begin{array}{l}\text { Structure/Formula } \\
\text { (oxidized form) }\end{array}$ & $\begin{array}{l}\text { Redox potential } \\
\text { (vs } \mathrm{Ag} / \mathrm{AgCl} \text { ) }\end{array}$ & $\begin{array}{c}\text { Charge } \\
\text { (Ox/Red) }\end{array}$ \\
\hline Potassium ferricyanide & $\begin{array}{l}\text { Small inorganic } \\
\text { metal complex }\end{array}$ & $\mathrm{K}_{3}\left[\mathrm{Fe}_{3}(\mathrm{CN})_{6}\right]$ & $+0.23 \mathrm{~V}^{(14)}$ & $-3 /-4$ \\
\hline $\begin{array}{l}\text { Hexaammineruthenium(III) } \\
\text { chloride }\end{array}$ & $\begin{array}{l}\text { Small inorganic } \\
\text { metal complex }\end{array}$ & {$\left[\mathrm{Ru}\left(\mathrm{NH}_{3}\right)_{6}\right] \mathrm{Cl}_{3}$} & $-0.11 \mathrm{~V}^{(15)}$ & $+3 /+2$ \\
\hline mPMS & $\begin{array}{c}\text { Small organic } \\
\text { compound } \\
\text { (phenazine derivative) }\end{array}$ & $\mathrm{H}_{3} \mathrm{CO}-\underset{\|}{\|}{ }_{\mathrm{S}}^{\mathrm{O}}-\mathrm{O}^{-}$ & $-0.14 \mathrm{~V}^{(16)}$ & $+/ 0$ \\
\hline
\end{tabular}

*oxidized form/reduced form 
that could be oxidized at the electrode. Although mPMS is more stable than PMS,${ }^{(16)} \mathrm{mPMS}$ is known to degrade in non-acidic solutions. ${ }^{(18)}$ Therefore, the interfering substance might be a degradation product. Another possibility is that a portion of the mPMS was in its reduced state.

With hexaammineruthenium(III), no response currents were obtained (Fig. 4). This indicates that $A v$ LOx does not utilize hexaammineruthenium(III) as an electron acceptor.

Whether a mediator can accept electrons from an enzyme should depend on the redox potential; mediators with a high redox potential should accept electrons more readily. Indeed, the mediator with the highest redox potential in this study, ferricyanide, does accept electrons readily from $A v$ LOx. However, although the redox potentials of mPMS and hexaammineruthenium(III) are similar, mPMS accepts electrons from $A v$ LOx, while hexaammineruthenium(III) does not. Nevertheless, the redox potential of hexaammineruthenium(III) should be high enough to accept electrons from the active center of $A v \mathrm{LOx}, \mathrm{FMN}$ (redox potential: $-0.42 \mathrm{vs} \mathrm{Ag} / \mathrm{AgCl}$ ). ${ }^{(19)}$ Thus, the redox potential does not explain the phenomena observed in this study.

The few previous reports on the comparison of mediators hint that factors other than the redox potential influence the suitability of a mediator. In 1988, Taniguchi et al. mentioned "some specific interaction", but did not go into more detail. ${ }^{(5)}$ Later, in 2001, Hirano et al. stated that the chemical structure of the mediator is crucial, but they, too, did not go into more detail. ${ }^{(11)}$ Finally, in 2013, Nieh et al. compared metal complexes bound to polymers and concluded that the electrostatic repulsion of negative ligands and the local surface charge of the enzyme, as well as steric hindrance, lead to less efficient mediators. ${ }^{(13)}$

The mediators used in this study are all small and of similar size, so steric hindrance does not explain any differences. Therefore, the most likely explanation is the electrostatic interaction of the mediators and the local surface charge of $A v \mathrm{LOx}$. It seems that, especially for small mediators with strong charges, the charge of the mediator and the sign of the charge have a great influence on its suitability as a mediator. The relatively uncharged mPMS is the most effective mediator for $A \nu \mathrm{LOx}$ in this study. The negatively charged ferricyanide does function as a mediator for $A v \mathrm{LOx}$; however, it is not as effective as mPMS. Therefore, $A v \mathrm{LOx}$ can tolerate negative charges fairly well, although the negative charge of the ferricyanide seems to hinder the electron transfer slightly, which is in accordance with the results of Nieh et al. ${ }^{(13)}$ The positively charged hexaammineruthenium(III) is not utilized as an electron acceptor by $A v$ LOx. This suggests that the access of the hexaammineruthenium(III) to the active center of $A v \mathrm{LOx}$ is blocked, despite the small size of the mediator.

\section{Conclusions}

In this study, the preferences of $A v \mathrm{LOx}$ for mediators that have a strong positive charge [hexaammineruthenium(III)], a strong negative charge (ferricyanide), or are relatively uncharged (mPMS) were investigated electrochemically. For this, recombinantly expressed $A v \mathrm{LOx}$ was used. The relatively uncharged mediator mPMS was most effective. With $100 \mathrm{mM}$ mPMS, near perfect linearity was achieved up to $50 \mathrm{mM}$ lactate. However, mPMS is known to be unstable, so that such high mPMS concentrations are impractical for commercial use. ${ }^{(18)}$ The negatively charged ferricyanide was less effective than mPMS but did successfully transfer electrons from $A v \mathrm{LOx}$ to the electrode. This suggests that $A v \mathrm{LO}$ tolerates negative charges fairly well. The linear range (up to $30 \mathrm{mM}$ ) and sensitivity $(0.64 \mu \mathrm{A} / \mathrm{mM}$ ) achieved with $100 \mathrm{mM}$ ferricyanide 
should be sufficient for blood lactate sensors (normal range $0.5-1.8 \mathrm{mM}$, maximal with exercise $25 \mathrm{mM}) .{ }^{(1,2)}$ Ferricyanide is already the most common mediator in commercial enzyme sensors. Finally, $A v$ LOx did not utilize the hexaammineruthenium(III) as an electron acceptor, suggesting that the access of the positively charged hexaammineruthenium(III) to the FMN in the active center of $A v \mathrm{LOx}$ is blocked by the protein scaffold. Therefore, hexaammineruthenium(III) cannot be used as the primary electron acceptor in enzyme sensor strips utilizing $A v L O x$. However, hexaammineruthenium(III) is the most desirable mediator for commercial use. Detailed structural investigations of $A v \mathrm{LOx}$ might reveal how the access of the hexaammineruthenium(III) to the active center is blocked, which might lead to strategies to engineer the enzyme to utilize hexaammineruthenium(III) as a mediator.

\section{Acknowledgments}

The authors thank i-SENS Inc. (Seoul, Republic of Korea) for their kindness in providing the SPCEs.

\section{References}

1 O. Kruse, N. Grunnet, and C. Barfod: Scand. J. Trauma Resuscit. Emerg. Med. 19 (2011) 74.

2 M. L. Goodwin, J. E. Harris, A. Hernández, and L. B. Gladden: J. Diabetes Sci. Technol. 1 (2007) 558.

3 K. Maeda-Yorita, K. Aki, H. Sagai, H. Misaki, and V. Massey: Biochimie 77 (1995) 631.

4 I. Taurino, R. Reiss, M. Richter, M. Fairhead, L. Thöny-Meyer, G. De Micheli, and S. Carrara: Electrochim. Acta 93 (2013) 72.

5 I. Taniguchi, S. Miyamoto, S. Tomimura, and F. M. Hawkridge: J. Electroanal. Chem. Interfacial Electrochem. 240 (1988) 333

6 J. Kulys, W. Schuhmann, and H.-L. Schmidt: Anal. Lett. 25 (1992) 1011.

7 K. Hirano, H. Yamato, K. Kunimoto, and M. Ohwa: Biosens. Bioelectron. 17 (2002) 315.

8 M. Boujtita, M. Chapleau, and N. E. Murr: Electroanalysis 8 (1996) 485.

9 Y. Kobayashi, T. Hoshi, and J. Anzai: Chem. Pharm. Bull. 49 (2001) 755.

10 K. Hirano, H. Yamato, K. Kunimoto, and M. Ohwa: Sens. Actuator, B 86 (2002) 88.

11 K. Hirano, H. Yamato, K. Kunimoto, and M. Ohwa: Bull. Chem. Soc. Jpn. 74 (2001) 2369.

12 H. Liu and J. Deng: Electrochim. Acta 40 (1995) 1845.

13 C.-H. Nieh, S. Tsujimura, O. Shirai, and K. Kano: J. Electroanal. Chem. 689 (2013) 26.

14 J. E. O’Reilly: Biochim. Biophys. Acta, Bioenerg. 292 (1973) 509.

15 G. Metzker, I. de Aguiar, S. C. Martins, M. S. Schultz, L. C. G. Vasconcellos, and D. W. Franco: Inorg. Chim. Acta 416 (2014) 142.

16 R. Hisada and T. Yagi: J. Biochem. 82 (1977) 1469.

17 https://www.pmda.go.jp/PmdaSearch/ivdDetail/ResultDataSetPDF/100639_25A2X00001000013_A_01_01 (accessed November 2017; in Japanese).

18 http://dominoweb.dojindo.co.jp/goodsr7.nsf/View_Display/M470?OpenDocument (accessed November 2017; in Japanese).

19 A. Okamoto, K. Hashimoto, K. H. Nealson, and R. Nakamura: Proc. Natl. Acad. Sci. 110 (2013) p. 7856. 


\section{About the Authors}

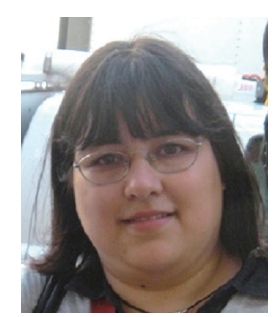

Noya Loew received her M.S. and Ph.D. degrees from the University of Potsdam, Germany, in 2003 and 2008, respectively. From 2008 to 2014, she was a postdoctoral researcher at Tokyo Institute of Technology, Japan. Since 2015, she has been a specially appointed assistant professor/postdoctoral researcher at Tokyo University of Agriculture and Technology. Her research interests are in biosensors, bioelectronics, and bioelectrochemistry.

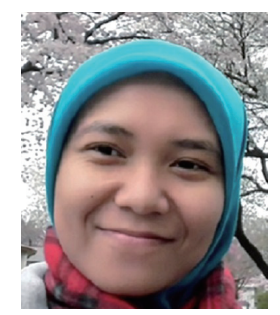

Maya Fitriana received her B.S. degree from the State University of Malang, Indonesia, in 2010. Since 2013, she has been an instructor at Sumbawa University of Technology, Indonesia. Since 2016, she has been a Ph.D. student at Tokyo University of Agriculture and Technology. Her research interests are in bioengineering, biomolecular engineering, and biosensors.

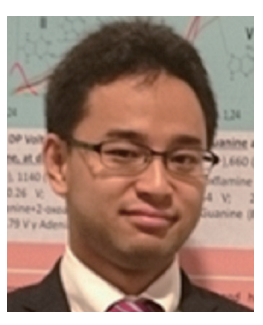

Kentaro Hiraka received his B.S. degree from Tokyo University of Agriculture and Technology, Japan, in 2016. Since 2016, he has been a master course student at Tokyo University of Agriculture and Technology. His research interests are in bioengineering, biomolecular engineering, and biosensors.

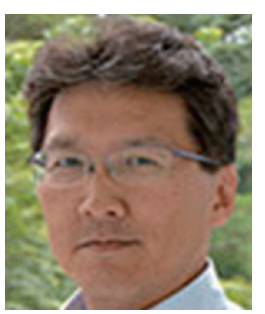

Koji Sode received his B.S., M.S., and Ph.D. degrees from the Tokyo Institute of Technology, Japan, in 1983, 1986, and 1988, respectively. Since 2000, he has been a professor at Tokyo University of Agriculture and Technology. Since 2003, he has been a science and technology advisor at Ultizyme International Ltd. His research interests are in bioengineering, biomolecular engineering, and biosensors.

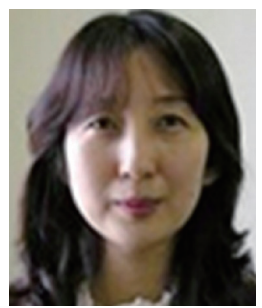

Wakako Tsugawa received her B.S. degree from the Tokyo Institute of Technology, Japan, in 1987 and her Ph.D. degree from Tokyo University of Agriculture and Technology, Japan, in 1998. Since 2005, she has been an associate professor at Tokyo University of Agriculture and Technology. Her research interests are in bioengineering, biomolecular engineering, and biosensors. 